\title{
Effect of Elevated Intracranial Pressure on Amplitudes and Frequency Tuning of Ocular Vestibular Evoked Myogenic Potentials Elicited by Bone-Conducted Vibration
}

\author{
Robert Gürkov, ${ }_{1}^{1}$ Guillaume Speierer, ${ }^{2}$ Luis Wittwer, ${ }^{2}$ and Roger Kalla ${ }^{2}$
}

\begin{abstract}
Objective: Recently, it could be demonstrated that an increased intracranial pressure causes a modulation of the air conducted sound evoked ocular vestibular evoked myogenic potential (oVEMP). The mechanism for this modulation is not resolved and may depend on a change of either receptor excitability or sound energy transmission.
\end{abstract}

Design: OVEMPs were elicited in 18 healthy subjects with a minishaker delivering 500 and $1000 \mathrm{~Hz}$ tone bursts, in supine and tilted positions.

Results: The study could confirm the frequency tuning of oVEMP. However, at neither stimulus frequency could a modulating effect of increased intracranial pressure be observed.

Conclusion: These data suggest that the observed modulation of the OVEMP response by an increased intracranial pressure is primarily due to the effect of an increased intralabyrinthine pressure onto the stiffness of the inner ear contents and the middle ear-inner ear junction. Future studies on the effect of intracranial pressure on oVEMP should use airconducted sound and not bone-conducted vibration.

Key words: Intracranial pressure, oVEMP, Vertigo.

(Ear \& Hearing 2016;XX;00-00)

\section{INTRODUCTION}

Recently, ocular vestibular evoked myogenic potentials (oVEMP) have emerged as a tool for assessment of utricular function. They are short-latency myogenic potentials which can be elicited in response to vestibular stimulation, for example, by air-conducted sound (ACS) or bone-conducted vibration (BCV; reviewed in Kantner \& Gürkov 2012). Otolithic afferent neurons trigger reflexive electromyographic activity of the extraocular muscles which can be recorded beneath the eye contralateral to the stimulated ear by use of surface electrodes. The pathway from the human otolith organs to the extraocular muscles reflects the vestibulo-ocular reflex (Iwasaki et al. 2007; Rosengren et al. 2010). The oVEMP waveform begins with a negative peak with a latency of about $10 \mathrm{msec}(\mathrm{n} 1)$, followed by a positive peak at about $15 \mathrm{msec}(\mathrm{p} 1)$. Although a minor saccular contribution to the oVEMP cannot be excluded, it represents mainly a utricular function (Curthoys et al. 2012).

The diagnostic usefulness of oVEMP has been confirmed for the superior canal dehiscence syndrome, an inner ear disorder characterized by hypersensitivity to sound and vertigo attacks elicited by sound stimulation (Tullio-phenomenon; Janky et al. 2013). Furthermore, the frequency tuning characteristics of ACS oVEMP are a promising new vestibular test to detect

${ }^{1}$ Department of Otolaryngology Head and Neck Surgery, German Center for Vertigo and Balance Disorders, University Hospital Munich, Campus Grosshadern, Munich, Germany; and ${ }^{2}$ Department of Neurology, Division of Cognitive and Restorative Neurology, University Hospital Bern, Bern, Switzerland endolymphatic hydrops in Menière's disease (Jerin et al. 2014). OVEMP responses are very sensitive to changes in the position of the eye ball (Kantner \& Gürkov 2014) and to changes of body position (Gürkov \& Kantner 2013). Recently, it could be demonstrated that an increased intracranial pressure (ICP) causes a modulation of the ACS evoked oVEMP (Jerin \& Gürkov 2014), predominantly at lower stimulus frequency (Jerin et al. 2015). While a modulation of auditory function by increased ICP has been known for otoacoustic emissions (Deppe et al. 2013), this was the first evidence of a vestibular function test to be modulated by ICP changes. However, it is not yet known whether the same effect would be observed when the oVEMP is not elicited by ACS but by BCV stimuli.

The mechanism for this modulation observed in ACS oVEMP may depend either on (1) a change in vestibular hair cell excitability by increased hydrostatic pressure (Düwel et al. 2003 ) or (2) on a change in sound energy transmission into the vestibulum by outward displacement of the stapes footplate, similar to the condition of endolymphatic hydrops in Menière's disease (Bouccara et al. 1998).

We therefore examined the effect of an elevated ICP condition on the oVEMP response to BCV stimuli. We hypothesized that the abovementioned mechanism (1) should also affect the BCV oVEMP, while mechanism (2) should not play a major role for the BCV oVEMP response during increased ICP.

\section{MATERIALS AND METHODS}

The study was conducted at a university medical centre and was approved by the local ethics committee (Protocol No. 215-13). All subjects gave informed consent to the study. The study population consisted of 18 healthy volunteers. Of these, oVEMP could reproducibly elicited in 15 subjects, 9 males and 6 females, age between 23 and 26 years, with no history of any audiovestibular disorder. Before testing, Weber and Rinne test as well as otoscopy were performed to exclude any conductive hearing loss. OVEMP were recorded using an Eclipse EP 25 evoked potential system (Interacoustics AS, Assens, Denmark). A hand-held electromechanical vibrator (Minishaker 4810, Brüel\&Kjaer, Denmark) fitted with a short bolt terminated in a plastic cap was used. The vibrator delivered a tone burst on the subject's skull at Fz (midline of the hairline). The operator held the vibrator by hand, supporting its weight. The static force resulting from the weight of the hand-held minishaker was $3 \mathrm{~N}$. Calibration was performed with an artificial mastoid (Bruel \& Kjaer Type 4930). Two stimulus frequencies were used: 500 and $1000 \mathrm{~Hz}$. The input signal was amplified by a custom amplifier (PA 2718, Brüel\&Kjaer, Denmark), the drive voltage was adjusted and fixed to produce a peak to peak force equivalent to $138 \mathrm{~dB}$ force level, with a measured force of $8.0 \mathrm{~N}$ 
TABLE 1. Effect of head-down tilt on bone-conducted vibration induced oVEMP

\begin{tabular}{|c|c|c|c|}
\hline Stimulus Frequency & \multicolumn{3}{|c|}{ Condition } \\
\hline $500 \mathrm{~Hz}$ & $23.04( \pm 9.83)$ & $23.13( \pm 10.4)$ & 0.72 \\
\hline$p$ value $(500 \mathrm{~Hz}$ vs. $1000 \mathrm{~Hz})$ & $<0.0001$ & $<0.0001$ & \\
\hline $1000 \mathrm{~Hz} / 500 \mathrm{~Hz}$ ratio & $0.55( \pm 0.17)$ & $0.57( \pm 0.13)$ & 0.42 \\
\hline
\end{tabular}

Results are shown as n10-p15 amplitudes in $\mu V$ (mean \pm SD). $p$ value obtained from Wilcoxon paired samples test.

OVEMP, ocular vestibular evoked myogenic potential.

and a reference force of $1 \mu \mathrm{N}$. Averaging of the responses to 100 stimulus repetitions, presented at a repetition rate of $5.1 / \mathrm{s}$, ensured sufficient reproducibility. The EMG was band passfiltered (1500 Hz to $3.3 \mathrm{~Hz}$ 6/oct) and recorded from $20 \mathrm{msec}$ before to $60 \mathrm{msec}$ after stimulus onset. The active electrode was positioned inferior to the lower-lid margin and the reference electrode was placed 2 to $3 \mathrm{~cm}$ below on the cheeks. Impedances were maintained below $3 \mathrm{k} \Omega$. The oVEMP amplitudes were defined as the peak to peak difference between the $\mathrm{n} 1$ and the $\mathrm{p} 2$ peaks. Subjects were in a supine position during the measurements. OVEMP were recorded in two conditions: (1) lying flat and (2) with the body elevated by 30 degrees with respect to the head, while maintaining a constant horizontal head orientation in both conditions, as previously described (Jerin \& Gürkov 2014). The tilt angle was controlled by an angle meter integrated
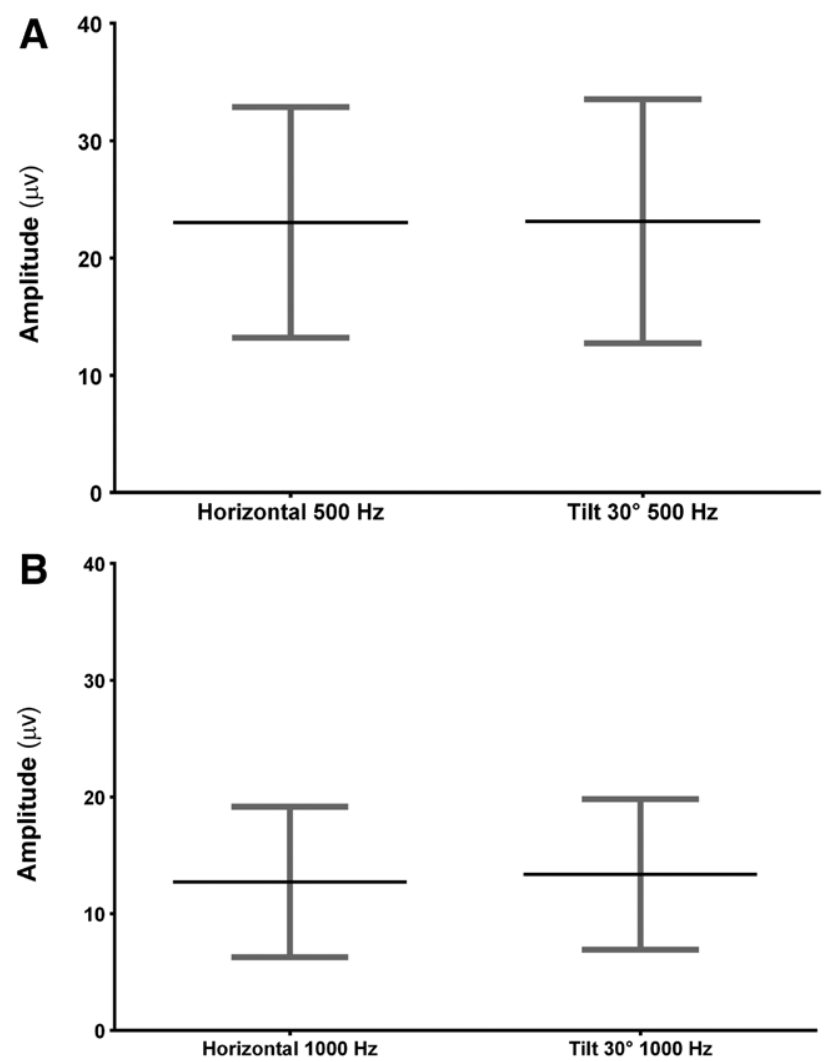

Fig. 1. A, Mean oVEMP amplitudes in $\mu \mathrm{V}( \pm \mathrm{SE})$ in 15 healthy subjects (30 ears), in response to $500 \mathrm{~Hz}$ bone-conducted vibration. B, Mean oVEMP amplitudes in $\mu \mathrm{V}( \pm \mathrm{SE})$ in 15 healthy subjects (30 ears), in response to 1000 $\mathrm{Hz}$ bone-conducted vibration. OVEMP, ocular vestibular evoked myogenic potential. into the tilt table. Based on the invasively measured data from Chapman et al. (1990), the dependence of the ICP on the tilt angle may be described as a linear function (ICP $=a(\sin \alpha-1) 2$ $-b$, with $\alpha=$ tilt angle from the horizontal, $a=10.5 \mathrm{~cm} \mathrm{H}_{2} \mathrm{O}$ and $b=0.81 \mathrm{~cm} \mathrm{H}_{2} \mathrm{O}$ ) in the range of 0 to 30 degrees (de Kleine et al. 2000). We therefore expected an approximate increase in ICP from 10 to $22 \mathrm{~cm} \mathrm{H}_{2} \mathrm{O}$ in response to the tilting maneuvre. Gaze position was controlled by a head-mounted laser beam gaze target, calibrated to an upgaze angle of 30 degrees, as previously described (Jerin et al. 2015). Pauses between single measurements and randomization of the sequence of single measurements were used to avoid confounding effects of fatigue. Data were analyzed with GraphPad PRISM software, using the nonparametric Wilcoxon test for paired samples. A $p$ value $<0.05$ was considered statistically significant.

\section{RESULTS}

Fifteen subjects had reproducible oVEMP responses to BCV stimulation. In the baseline condition, the mean oVEMP amplitudes were $23.04 \mu \mathrm{V}$ for the $500 \mathrm{~Hz}$ stimulus and $12.72 \mu \mathrm{V}$ for the $1000 \mathrm{~Hz}$ stimulus. In the 30-degree tilt condition, the mean oVEMP amplitudes remained stable at 23.13 and $13.37 \mu \mathrm{V}$ for 500 and $1000 \mathrm{~Hz}$, respectively (Table 1). Figure 1A shows oVEMP amplitudes $( \pm$ SE) for the $500 \mathrm{~Hz}$ stimulus, Figure 1B shows oVEMP amplitudes $( \pm \mathrm{SE})$ for the $1000 \mathrm{~Hz}$ stimulus. A representative example waveform of $\mathrm{BCV}$ and ACS oVEMP is shown in Figure 2.

\section{DISCUSSION}

Our results stand in contrast to the results obtained by a previous study with identical experimental tilting maneuvre (Jerin \& Gürkov 2014) in a different study population by eliciting oVEMP with $500 \mathrm{~Hz}$ tone burst ACS during resting state and during elevated ICP. They found that increasing ICP significantly and linearly reduces the oVEMP response to ACS. In their study, a tilt angle of 30 degrees leads to a decrease in $500 \mathrm{~Hz}$ ACS oVEMP amplitudes from 4.59 to $3.25 \mu \mathrm{V}$, corresponding to a $29 \%$ change. In accordance with previous studies on BCV oVEMP, the present data confirm the observed frequency tuning with the responses to $500 \mathrm{~Hz}$ stimuli resulting in about 1.8 times larger amplitudes than the responses to $1000 \mathrm{~Hz}$ stimuli.

However, in the present study, the BCV elicited responses to both frequencies, 500 and $1000 \mathrm{~Hz}$, were not altered by the tilting maneuvre. The frequency tuning of BCV induced oVEMP, calculated as the ratio of the $500 \mathrm{~Hz} / 1000 \mathrm{~Hz}$ responses was also unchanged by the tilting maneuvre. Therefore, the previously documented effect of increased ICP on the frequency 

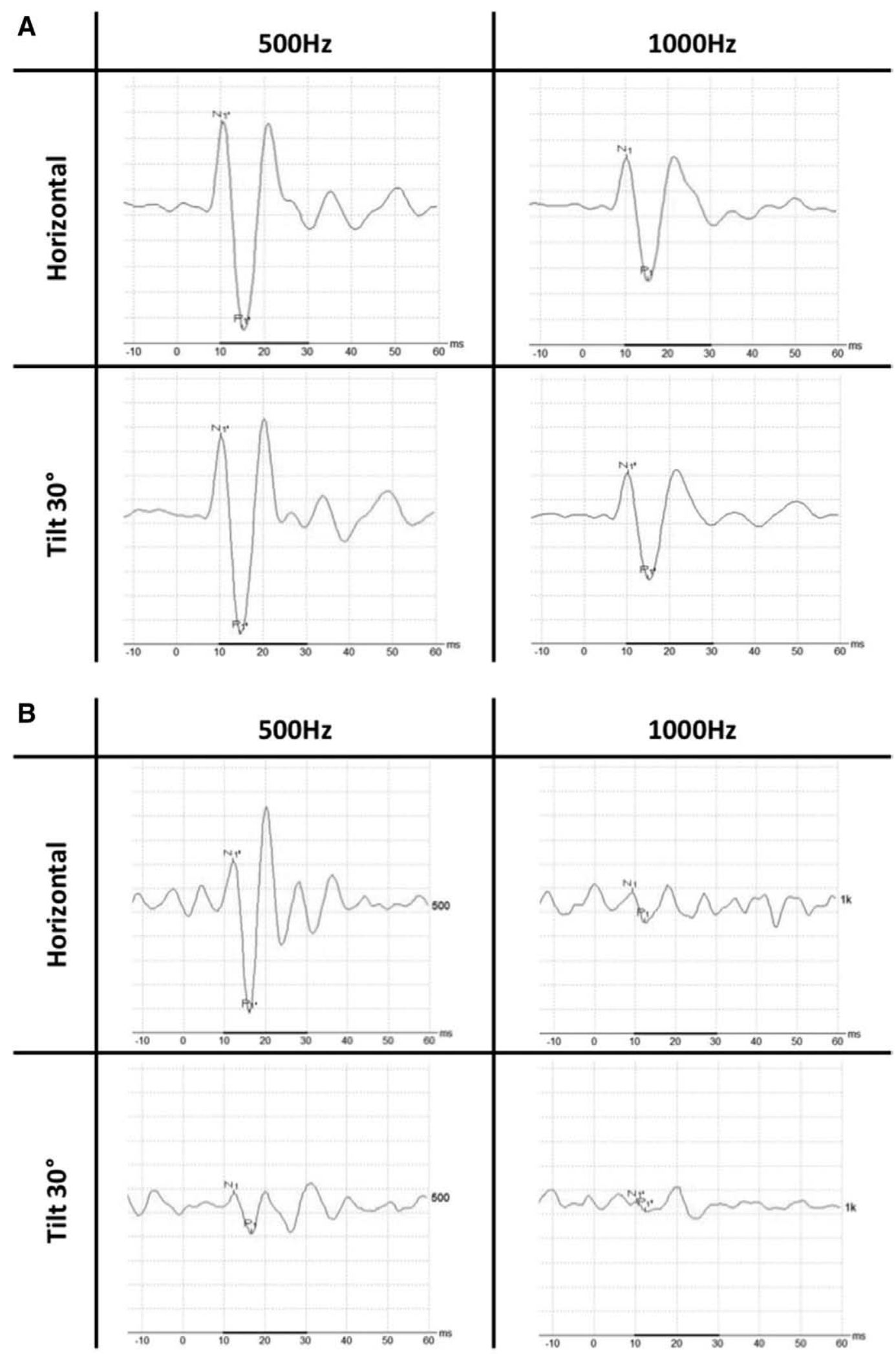

Fig. 2. A, Example oVEMP waveform from one healthy subject, in response to 500 and $1000 \mathrm{~Hz}$ bone-conducted vibration. B, Example oVEMP waveform from one healthy subject, in response to 500 and $1000 \mathrm{~Hz}$ air-conducted sound. OVEMP, ocular vestibular evoked myogenic potential.

tuning of the ACS elicited oVEMP response (Jerin et al. 2015) cannot be observed for BCV-elicited oVEMP. In concordance with the present study, a recent report comparing ACS and BCV stimuli at $500 \mathrm{~Hz}$ for their dependence on ICP changes found that $500 \mathrm{~Hz}$ ACS oVEMP are modulated by ICP, but $500 \mathrm{~Hz}$ BCV oVEMP are not (Gürkov et al. 2016). 
An increase in ICP is transmitted to the inner ear labyrinth via the communication of the CSF space with the endolymphatic and perilymphatic spaces of the inner ear, the cochlear, and vestibular aqueducts. Otoacoustic emission recordings have shown that this equilibration takes approximately $30 \mathrm{sec}$ (de Kleine et al. 2000). This functional connection between the CSF space and the labyrinth is thought to account for the various audiovestibular symptoms which occur in patients with elevated CSF pressure (e.g., idiopathic intracranial hypertension) and patients with abnormal patency of these communications (e.g., large vestibular aqueduct syndrome). The effect of increased ICP on the oVEMP response is in principle suitable as a basis to develop a VEMP-based method of noninvasively monitoring ICP over time in individual patients. It may also be diagnostically useful in disorders with a "third window lesion" between the labyrinth and CSF space, for example, superior canal dehiscence syndrome. Janky et al. (2015) addressed this possibility, and they could not find an additional diagnostic value for the tilttable oVEMP measurement. However, they used a different experimental set-up where the tilt maneuver comprised the whole body including the head and therefore changed the gravitoinertial orientation of the vestibular sensory macula; therefore, their data cannot be directly compared with the present study.

Taylor et al. (2014) explored effects of posture on oVEMP in a different set-up: They changed whole body/head position by stepwise moving the subjects within the roll plane around a full 360-degree rotation. This procedure led to a depression of both ACS and BCV oVEMP when the roll tilt angle exceeded 90 degree, that is, when the head was reaching an orientation below the horizontal. While this procedure also leads to increased ICP, the procedural differences prevent a direct comparison of these data with our data: Besides the use of different stimulus (broad spectrum click), their positioning maneuvre not only changed ICP but also the spatial orientation of the head and the otolithic membranes with respect to gravity. A possible explanation that Taylor et al. offered, and that could explain this difference, is a possible modulatory effect of saccular afferents on the utricular afferents at the level of the vestibular nucleus, given that the head-down maneuvre of Taylor et al. gradually aligns the vertically oriented saccular macula with the force of gravity where the mechanical loading on saccular hair cells is maximal, thereby potentially reducing the capacity for further hair cell displacement by the stimulus.

Yet another maneuvre was used by Wang et al. (2014) to explore postural effects on oVEMP: They elicited ACS and BCV oVEMP in the sitting, the supine, and the supine-headhanging (neck extended) positions. The only significant difference in this set-up was between sitting and head-hanging positions for the BCV oVEMP, with the latter position yielding slightly larger amplitudes than the former (mean 15.9 versus $13.8 \mu \mathrm{V})$. Again this procedure induced changes of both parameters, the spatial orientation of the head and the elevation of the head relative to the body. In contrast, our set-up was designed to keep the spatial orientation of the head constant and to increase the ICP by only changing the relative elevation of the head versus the body.

In summary, our data suggest that the observed modulation of the oVEMP response by an increased ICP is primarily due to the effect of an increased ILP onto the stapes footplate and an increased stiffness of the inner ear contents and the middle ear-inner ear junction. It is rather unlikely that the effect is mediated via a pressure-dependent change of vestibular receptor excitability, because this would be expected to occur during stimulation with both ACS and BCV. Furthermore, these results suggest that future studies on the effect of ICP on oVEMP should use ACS and not BCV as a stimulus to evoke oVEMP.

\section{ACKNOWLEDGMENTS}

We thank Dr. Georgios Mantokoudis and Dr. Claudia Jerin for helpful discussions.

The study was supported by the Volkswagen Stiftung.

The authors have no conflicts of interest to disclose.

Address for correspondence: Robert Gürkov, Department of Otolaryngology Head and Neck Surgery, University of Munich, Grosshadern Medical Center, Marchioninistr. 15, 81377 Munich, Germany. E-mail: robert. guerkov@med.uni-muenchen.de

Received November 28, 2015; accepted March 29, 2016.

\section{REFERENCES}

Bouccara, D., Ferrary, E., El Garem, H., et al. (1998). Inner ear pressure in Menière's disease and fluctuating hearing loss determined by tympanic membrane displacement analysis. Audiology, 37, 255-261.

Chapman, P. H., Cosman, E. R., Arnold, M. A. (1990). The relationship between ventricular fluid pressure and body position in normal subjects and subjects with shunts: A telemetric study. Neurosurgery, 26, 181-189.

Curthoys, I. S., Vulovic, V., Sokolic, L., et al. (2012). Irregular primary otolith afferents from the guinea pig utricular and saccular maculae respond to both bone conducted vibration and to air conducted sound. Brain Res Bull, 89, 16-21.

de Kleine, E., Wit, H. P., van Dijk, P., et al. (2000). The behavior of spontaneous otoacoustic emissions during and after postural changes. J Acoust Soc Am, 107, 3308-3316.

Deppe, C., Kummer, P., Gürkov, R., et al. (2013). Influence of the individual DPOAE growth behavior on DPOAE level variations caused by conductive hearing loss and elevated intracranial pressure. Ear Hear, 34, $122-131$.

Düwel, P., Jüngling, E., Westhofen, M., et al. (2003). Potassium currents in vestibular type II hair cells activated by hydrostatic pressure. Neuroscience, 116, 963-972.

Gürkov, R., \& Kantner, C. (2013). Modulation of oVEMP amplitudes by lateral head tilts. Clin Neurophysiol, 124, 1911-1912.

Gürkov, R., Speierer, G. Wittwer, L., et al. (2016). Differential effect of elevated intralabyrinthine pressure on ocular vestibular evoked myogenic potentials elicited by air conducted sound and bone conducted vibration. Clin Neurophysiol, 127: 2115-2118.

Iwasaki, S., McGarvie, L. A., Halmagyi, G. M., et al. (2007). Head taps evoke a crossed vestibulo-ocular reflex. Neurology, 68, 1227-1229.

Janky, K. L., Nguyen, K. D., Welgampola, M., et al. (2013). Air-conducted oVEMPs provide the best separation between intact and superior canal dehiscent labyrinths. Otol Neurotol, 34, 127-134.

Janky, K. L., Zuniga, M. G., Schubert, M. C., et al. (2015). The effect of increased intracranial pressure on vestibular evoked myogenic potentials in superior canal dehiscence syndrome. Clin Neurophysiol, 126, 780-786.

Jerin, C., \& Gürkov, R. (2014). Posture-induced changes of ocular vestibular evoked myogenic potentials suggest a modulation by intracranial pressure. Exp Brain Res, 232, 2273-2279.

Jerin, C., Bartl, K., Schneider, E., et al. (2015). Enhancing the reproducibility of ocular vestibular evoked myogenic potentials by use of a visual target originating from a head-mounted laser. Eur Arch Otorhinolaryngol, 272, 2737-2740.

Jerin, C., Berman, A., Krause, E., et al. (2014). Ocular vestibular evoked myogenic potential frequency tuning in certain Menière's disease. Hear Res, 310, 54-59. 
Jerin, C., Wakili, R., Kalla, R., et al. (2015). The effect of increasing intracranial pressure on ocular vestibular-evoked myogenic potential frequency tuning. Ear Hear, 36, e336-e341.

Kantner, C., \& Gürkov, R. (2012). Characteristics and clinical applications of ocular vestibular evoked myogenic potentials. Hear Res, 294, $55-63$.

Kantner, C., \& Gürkov, R. (2014). The effects of commonly used upward gaze angles on ocular vestibular evoked myogenic potentials. Otol Neurotol, 35, 289-293.
Rosengren, S. M., Welgampola, M. S., Colebatch, J. G. (2010). Vestibular evoked myogenic potentials: Past, present and future. Clin Neurophysiol, $121,636-651$.

Taylor, R. L., Xing, M., Black, D. A., et al. (2014). Ocular vestibular evoked myogenic potentials: The effect of head and body tilt in the roll plane. Clin Neurophysiol, 125, 627-634.

Wang, S. J., Tseng, C. C., Young, Y. H. (2014). Selective effects of head posture on ocular vestibular-evoked myogenic potential (oVEMP) by bone-conducted vibration. Clin Neurophysiol, 125, 621-626. 